\title{
Expression and clinical significance of extracellular matrix metalloproteinase inducer, EMMPRIN/CD147, in human osteosarcoma
}

\author{
QIANG LU ${ }^{1}$, GANG LV ${ }^{1}$, ANDRE KIM ${ }^{2}$, JONG-MYUNG HA ${ }^{2}$ and SUHKMAN KIM $^{3}$ \\ ${ }^{1}$ Department of Orthopaedics, The First Affiliated Hospital of China Medical University, \\ Shenyang, Liaoning 110001, P.R. China; \\ ${ }^{2}$ Department of Pharmaceutical Engineering, College of Medical Life Sciences, Silla University, Busan 617-736; \\ ${ }^{3}$ Department of Chemistry and Chemistry Institute for Functional Materials, Pusan National University, \\ Busan 609-735, Republic of Korea
}

Received April 16, 2012; Accepted July 26, 2012

DOI: $10.3892 / \mathrm{ol} .2012 .981$

\begin{abstract}
Osteosarcoma is the most common primary malignant bone tumor in children and adolescents. Recent studies have shown that extracellular matrix metalloproteinase inducer (EMMPRIN/CD147) promotes adhesion, invasion and metastasis of malignant tumor cells. The aim of this study was to investigate the impact of EMMPRIN/CD147 expression on prognosis and its correlation with clinicopathological characteristics in patients with osteosarcoma. The expression of EMMPRIN/CD147 in 55 surgical specimens from patients with osteosarcoma at stage IIA or above, 15 non-tumor rib bone tissues, three human osteosarcoma cell lines (Saos-2, U-2OS and MG-63), the human osteoblast cell line HOB and the malignant melanoma cell line A375 were examined by immunohistochemistry, western blot analysis and ELISA, respectively. The potential association of the levels of EMMPRIN/CD147 expression in osteosarcoma specimens with the overall survival of patients was statistically analyzed. We found that the EMMPRIN/CD147 was expressed in 45 out of 55 osteosarcomas, with immunoreactivity primarily within the membrane and cytoplasm of tumor cells, but not in the non-tumor bone tissues. We also observed that EMMPRIN/CD147 was expressed in Saos-2, U-2OS, MG-63 and A375, but not in HOB cells. The levels of EMMPRIN/CD147 expression correlated positively with the pathological degree of osteosarcoma and negatively with the survival period of patients with osteosarcoma. The expression of EMMPRIN/CD147 is a potential factor in the development
\end{abstract}

Correspondence to: Professor Gang Lv, Department of Orthopaedics, The First Affiliated Hospital of China Medical University, No. 155 Nanjing North Street, Heping, Shenyang, Liaoning 110001, P.R. China

E-mail:lvgang09@gmail.com

Key words: extracellular matrix metalloproteinase inducer, osteosarcoma, prognosis, overall survival and prognosis of osteosarcoma and may be a novel therapeutic target of human osteosarcoma.

\section{Introduction}

Osteosarcoma arises from mesenchymal tissues and is the most common primary malignant bone tumor in children and adolescents $(1,2)$. Since osteosarcoma is highly aggressive and commonly metastasizes to the lung, patients with metastatic or recurrent osteosarcoma usually have an extremely poor prognosis (2-5). Although the diagnosis and treatment of osteosarcoma have been improved significantly during the past 30 years $(3,6)$, approximately $30-40 \%$ of patients experience osteosarcoma relapse, mostly within the first three years after diagnosis. Common factors, such as demographics (age and gender), tumor size, site and stage have been used for the prognosis of advanced osteosarcoma, but the results are difficult to reproduce due to the usage of various techniques and units for measurement. Furthermore, tumor-related metastasis and chemotherapeutic response are significant prognostic factors, but they usually occur at the late stage of osteosarcoma (1-4). Therefore, there is an urgent need for the discovery of new reliable and efficient biomarkers for the prognosis of osteosarcoma.

Extracellular matrix metalloproteinase inducer (EMMPRIN, also known as CD147, EMMPRIN/CD147) is a highly glycosylated transmembrane protein and is abundantly expressed on the membrane surface of various tumor cells, including non-small cell lung cancer (NSCLC) (7), macrophage-like lymphoid neoplasm P388D1 cells (8), thyroid carcinoma (9), primary cutaneous malignant melanoma (10), intrahepatic cholangiocarcinoma (11), colorectal/gastric cancer (12), renal cell carcinoma (13), prostate cancer (14), cervical cancer (15), epithelial ovarian cancer (16) and breast carcinoma (17). EMMPRIN/CD147 promotes survival, invasion and metastasis of tumor cells through multiple pathways and mechanisms, including the functional loss of p53, a tumor suppressor (18), upregulated expression of vascular endothelial growth factor (VEGF) $(13,19-21)$, disruption of 
transforming growth factor- $\beta 1$ (TGF- $\beta 1$ ), a growth-modulating factor (22), and regulation of the urokinase-type plasminogen activation (UPA) system of serine proteases (23). Therefore, EMMPRIN/CD147 has been suggested potentially as a prognostic biomarker for certain types of tumors and as a therapeutic target (24). However, the prognostic value of EMMPRIN/CD147 in human osteosarcoma remains to be elucidated.

The aim of present study was to examine whether EMMPRIN/CD147 could be expressed in osteosarcoma tissues and to analyze the potential association of the levels of EMMPRIN/CD147 expression with clinicopathological characteristics and survival outcome in Chinese patients with osteosarcoma.

\section{Materials and methods}

Cell lines and cultures. Human osteosarcoma cell lines (Saos-2, U-2OS and MG-63), human malignant melanoma cell line A375 and human osteoblast cell line Hob were obtained from the American Type Culture Collection (ATCC; Manassas, VA, USA) and cultured in Iscove's modified Dulbecco's medium (IMDM; Invitrogen, Carlsbad, CA, USA), supplemented with $20 \mathrm{U} / \mathrm{ml}$ penicillin, $100 \mu \mathrm{g} / \mathrm{ml}$ streptomycin (Sigma, Beijing, China), and 10\% heat-inactivated fetal bovine serum (FBS; Biowhittaker, Walkersville, $\mathrm{MD}, \mathrm{USA})$ at $37^{\circ} \mathrm{C}$ in a humidified incubator supplied with $5 \% \mathrm{CO}_{2}(25)$.

Western blot analysis. Western blot analysis was performed as described previously (26). Briefly, individual osteosarcoma cell lines grown to confluency were harvested and lysed in buffer containing $50 \mathrm{mmol} / \mathrm{l}$ Tris- $\mathrm{HCl}(\mathrm{pH} 8.0), 150 \mathrm{mmol} / \mathrm{l}$ $\mathrm{NaCl}, 100 \mu \mathrm{g} / \mathrm{ml}$ phenylmethan-sulfonylfluoride and $1 \%$ Triton X-100 for $30 \mathrm{~min}$ on ice, followed by centrifugation. After quantification of protein concentrations, equal amounts $(50 \mu \mathrm{g} / \mathrm{lane})$ of cell lysates were separated by $12 \%$ sodium dodecyl sulfate polyacrylamide gel electrophoresis (SDS-PAGE) and transferred to a nitrocellulose membrane. Subsequently, the membrane was blocked with $5 \%$ non-fat dry milk in TBST $[10 \mathrm{mmol} / 1$ Tris- $\mathrm{HCl}(\mathrm{pH} 7.5)$, $150 \mathrm{mmol} / \mathrm{l} \mathrm{NaCl}$ and $0.05 \%$ Tween-20] at room temperature for $1 \mathrm{~h}$. The proteins were probed with rabbit anti-human EMMPRIN/CD147 antibodies (1:1,000; Boster, Wuhan, China) or control anti-glyceraldehyde-3-phosphate dehydrogenase antibodies (anti-GAPDH, Sigma) at $4^{\circ} \mathrm{C}$ overnight. The bound antibodies were detected with horseradish peroxidase (HRP)-conjugated goat anti-rabbit antibodies (1:10,000; Boster) and visualized using the SuperSignal West Femto trial kit (Pierce Biotechnology, Rockford, IL, USA) (27), according to the manufacturer's instructions.

ELISA. Human osteosarcoma cell lines (Saos-2, U-2OS and MG-63), human malignant melanoma cell line A375 and human osteoblast cell line Hob were harvested and after being centrifuged, cell pellets were weighed and re-suspended in an equal volume of lysis buffer. Following centrifugation, the concentrations of EMMPRIN/CD147 in the supernatants were determined by ELISA using the EMMPRIN/CD147 ELISA kit, according to the manufacturer's instructions (Biosensis,
Thebarton, Australia), as reported in a previous study (28). The concentrations of cellular EMMPRIN/CD147 were determined according to the standard curve and expressed as $\mu \mathrm{g} / \mathrm{mg}$ wet cells.

Research subjects. Written informed consent was obtained from individual participants and the experimental protocol was approved by the Institution Research Board of The First Affiliated Hospital of China Medical University (Liaoning, China). A total of 55 patients with osteosarcoma were collected from the in-patient service at the Department of Orthopaedics (The First Affiliated Hospital of China Medical University) between 1997 and 2003 for this retrospective study. These patients had been diagnosed with osteosarcoma at stage IIA or above, according to the clinicopathological classification of diagnostic standards $(29,30)$. The patients were intravenously administered neoadjuvant chemotherapy of one cycle of $8 \mathrm{~g} / \mathrm{m}^{2}$ methotrexate for one day, and $120 \mathrm{mg} / \mathrm{m}^{2}$ doxorubicin and $75 \mathrm{mg} / \mathrm{m}^{2}$ cisplatin daily for three consecutive days for two cycles consecutively. Subsequently, these patients were subjected to surgical resection of the tumor, and their surgical margins were classified, according to the Enneking scoring system, as highly malignant intracompartmental osteogenic sarcomas (IIA), extracompartmental lesions (IIB) or osteogenic sarcomas with manifestation of metastases (III) $(29,30)$. Following surgery, specimens were collected from individual patients and evaluated for their diagnosis and adequacy of the surgical margins. Surgical resection of a tumor with radical or wide margins was considered as adequate, whereas those with marginal or intralesional margins were classified as inadequate. The patients received postoperative chemotherapy with two cycles of methotrexate, doxorubicin and cisplatin, as described above, while 35 patients with a poor histological response to preoperative chemotherapy, based on the percentage of tumor cell necrosis in the surgical specimens (31), were treated with the medicines described above and two cycles of $15 \mathrm{~g} / \mathrm{m}^{2}$ ifosfamide daily for five consecutive days. Patients were followed up and clinical data were updated for more than 5 years. Tumor recurrence at any site or mortality were defined as an adverse event. The clinicopathological features of the osteosarcoma patients are shown in Table I.

Immunohistochemistry. The cellular expression of EMMPRIN/CD147 in surgical tumor tissues from patients was detected by immunohistochemistry analysis, as described previously $(32,33)$ using the Streptavidin-Avidin-BiotinPeroxidase Complex (SABC). The specimen tissue sections at $4 \mu \mathrm{m}$ were treated with $3 \% \mathrm{H}_{2} \mathrm{O}_{2}$ for $10 \mathrm{~min}$ at room temperature. The sections were blocked with 5\% bovine serum albumin (Zhongshan, Beijing, China) in PBS solution for $20 \mathrm{~min}$ and probed with rabbit anti-human EMMPRIN/CD147 antibodies (1:300; Boster) at $4^{\circ} \mathrm{C}$ for $12 \mathrm{~h}$. After washing, the bound antibodies were detected with biotinylated goat anti-rabbit $\operatorname{IgG}(1: 100)$ and $\mathrm{SABC}$ complex at $30^{\circ} \mathrm{C}$ for $20 \mathrm{~min}$. The immunoreactivity was visualized by 3,3-diaminobenzidine tetrachloride (DAB) and counterstained with hematoxylin, then examined under a light microscope (CX41; Olympus, Tokyo, Japan) at x400 magnification. In addition, the formalin-fixed, paraffin-embedded tumor sections at $4 \mu \mathrm{m}$ were stained with hematoxylin and eosin (H\&E) for histological diagnosis. 
Table I. Correlation between EMMPRIN/CD147 expression and clinicopathological characteristics.

\begin{tabular}{|c|c|c|c|c|}
\hline \multirow[b]{2}{*}{ Parameter } & \multirow[b]{2}{*}{$\mathrm{n}(\%)$} & \multicolumn{2}{|c|}{ EMMPRIN/CD147 } & \multirow[b]{2}{*}{ P-value } \\
\hline & & Negative/weak & Moderate/strong & \\
\hline Total & $55(100)$ & 25 & 30 & \\
\hline Gender & & & & 0.162 \\
\hline Male & $32(58)$ & 12 & 20 & \\
\hline Female & $23(42)$ & 13 & 10 & \\
\hline Age (years) & & & & 0.495 \\
\hline$\leq 18$ & $37(65)$ & 18 & 19 & \\
\hline$>18$ & $18(35)$ & 7 & 11 & \\
\hline Anatomical location & & & & 0.539 \\
\hline Tibia/femur & $35(67)$ & 17 & 18 & \\
\hline Elsewhere & $20(33)$ & 8 & 12 & \\
\hline Tumor size $\left(\mathrm{cm}^{3}\right)$ & & & & 0.637 \\
\hline$\leq 50$ & $37(31)$ & 16 & 21 & \\
\hline$>50$ & $18(69)$ & 9 & 9 & \\
\hline Histological subtype & & & & 0.451 \\
\hline Osteoblastic & $25(45)$ & 9 & 16 & \\
\hline Chondroblastic & $20(24)$ & 10 & 10 & \\
\hline Fibroblastic & $9(29)$ & 5 & 4 & \\
\hline Not specified & $1(2)$ & 1 & 0 & \\
\hline Pathological classification & & & & $0.003^{\mathrm{a}}$ \\
\hline IIA & $18(33)$ & 14 & 4 & \\
\hline IIB & $20(36)$ & 7 & 13 & \\
\hline III & $17(31)$ & 4 & 13 & \\
\hline Treatment & & & & 0.580 \\
\hline Limb salvage & $33(64)$ & 14 & 19 & \\
\hline Amputation & $22(36)$ & 11 & 11 & \\
\hline Percentage of dead cells & & & & 0.001 \\
\hline$<90 \%$ & $35(67)$ & 10 & 25 & \\
\hline$\geq 90 \%$ & $20(33)$ & 15 & 5 & \\
\hline Surgical margins & & & & 0.707 \\
\hline Adequate & $53(96)$ & 24 & 19 & \\
\hline Inadequate & $2(4)$ & 1 & 1 & \\
\hline
\end{tabular}

${ }^{\mathrm{a}} \mathrm{P}<0.05$.

The negative controls for immunohistochemistry included using PBS control (without the primary EMMPRIN/CD147 antibody), and 15 non-tumor rib bone tissue samples from patients who had undergone surgery. As a positive control for CD147 expression, immunostaining was performed on a sample of prostate cancer tissue with known strong expression of CD147, which had been used in a previous study (34). The quality of staining was evaluated using consecutive control sections. The cells stained positively by anti-EMMPRIN/CD147 were assessed, as previously reported $(35,36)$. Briefly, the number of positively stained cells and total number of cells in a given area were evaluated by two pathologists in a blinded manner. If the amount of positively stained cells was $\leq 5 \%$ the tissue was considered as negative (-); 6-25\%, weak (+); 26-50\%, moderate (++); and $\geq 51 \%$, strong (+++). A total of at least 400 cells from five areas of individual tissue samples were evaluated.

Survival analysis. The overall survival of individual patients was defined from the day of surgery up to the last follow-up examination or death of the patient. Data from patients who had survived at the end of observation period were censored at their last follow-up visit. Individuals who succumbed to other diseases unrelated to osteosarcoma or survived at the end of the observation period were considered a censoring event. Event-free survival was calculated from the date of initial diagnosis. Overall survival and event-free survival curves were plotted for each group of patients, according to the levels of EMMPRIN/CD147 expression in the surgical specimen tissues. 
Statistical analysis. Data shown are the real case number and percentage for each group. All of the patients were stratified according to individual parameters, and the difference in the frequency of cases between groups was calculated by the $\chi^{2}$ test or Fisher's exact test. The difference in overall and event-free survival of each group of patients was analyzed by the log-rank test and the mean survival time between groups was calculated by Student's t-test, followed by analysis of the 95\% confidence interval (CI). The Cox proportional hazards model was used for multivariate analysis. A value of $\mathrm{P}<0.05$ was considered to indicate a statistically significant difference.

\section{Results}

EMMPRIN/CD147 is expressed by human osteosarcoma cell lines. The human osteosarcoma cells lines, Saos-2, U-2OS and MG-63, and human malignant melanoma cell line, A375, as well as human non-tumor osteoblast cell line, Hob, were cultured in vitro and harvested. After lysis, the cell lysates were separated by SDS-PAGE and the expression of EMMPRIN/CD147 was characterized by western blot assays (Fig. 1). There was no detectable EMMPRIN/CD147 expression in human non-tumor osteoblast cell line, Hob (data not shown). However, high levels of EMMPRIN/CD147 expression were observed in all of the three osteosarcoma cell lines tested and human malignant melanoma cell line, A375, and the relative levels of EMMPRIN/CD147 to the control GAPDH were undistinguishable among these cell lines. Furthermore, the ELISA analysis also indicated that EMMPRIN/CD17 was expressed in the osteosarcoma cells and malignant melanoma cell line A375 (Fig. 2). These data demonstrated that the EMMPRIN/CD147 was highly expressed in human osteosarcoma cells.

\section{EMMPRIN/CD147 is expressed in human osteosarcoma tumor} tissues. Next, we examined whether EMMPRIN/CD147 was expressed in spontaneously developed human osteosarcoma tissues. Specimens from 55 patients with osteosarcoma were collected and were defined as osteoblastic (25/55; Fig. 3A), chondroblastic (20/55; Fig. 3B), fibroblastic (9/55; Fig. 3C), and non-specific osteosarcoma (1/55), based on histological examinations. The demographic and clinicopathogenic characteristics are presented in Table I. Pathological classification identified those patients with osteosarcoma at stage IIA or above. These osteosarcomas were characterized by the presence of osteoid (bone formation) within the tumor tissues. The tumor cells were very polymorphic (anaplastic), similar to giant cells with numerous atypical mitoses or multinucleated osteoclast-like giant cells (Fig. 3A-C), similar to those observed in previous reports $(29,30)$.

Immunohistochemical analyses revealed that 45 of the 55 osteosarcoma tissue samples were positive for anti-EMMPRIN/CD147 staining (Fig. 4A-C), but negative in 10 out of 55 osteosarcoma tissue samples and negative controls (Fig. 4D and E) as well as non-tumor rib bone tissue samples (Fig. 4F). The relative levels of EMMPRIN/CD147 expression in the osteosarcoma tissues were classified as negative/weak $(25 / 55)$ or moderate/high (30/55), respectively (Table I). Furthermore, immunoreactivity against EMMPRIN/CD147 was observed predominantly in the membrane and cytoplasm of osteosarcoma tumor cells, but not in the nucleus of tumor

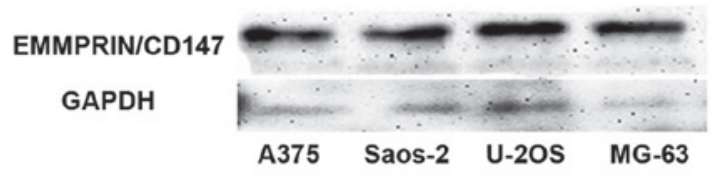

Figure 1. Western blot analysis of EMMPRIN/CD147 expression in osteosarcoma cell lines. A375, Saos-2, MG-63 and U-2OS cells were harvested and lyzed. Subsequently, the relative levels of EMMPRIN/CD147 expression in individual cell lines were characterized by western blot analysis using the anti-EMMPRIN/CD147 and anti-GAPDH antibodies, respectively. Data shown are representative images of individual cell lines from three separate experiments.

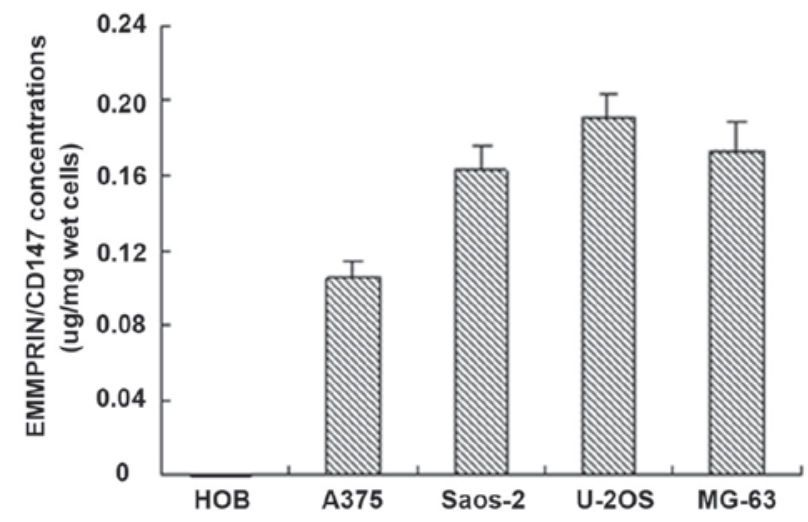

Figure 2. EMMPRIN/CD147 levels expressed by individual cell lines. Saos-2, U-2OS, MG-63, A375 and Hob cells were cultured and harvested when they reached confluency. The concentrations of cellular EMMPRIN/CD147 in individual cells were determined by ELISA using the EMMPRIN/CD147 ELISA kit. Data are expressed as means \pm SD of the EMMPRIN/CD147 concentrations per $\mathrm{mg}$ wet cells of individual cell lines from three separate experiments.

cells or in surrounding stromal cells (Fig. 4A-C). In addition, the intensity of anti-EMMPRIN/CD147 staining was notably higher in osteosarcoma cells than in the bone giant cell tumors and non-cancerous adjacent tissues. Notably, stratification of each measure indicated that the EMMPRIN/CD147 immunostaining intensity was closely associated with the pathological degree. Indeed, 13 out of 17 or 20 patients with osteosarcoma at stage III or IIB had strong staining of anti-EMMPRIN/CD147, while only four out of 18 patients with osteosarcoma at stage IIA were strongly positive for anti-EMMPRIN/CD147 $(\mathrm{P}<0.05$; Table I). However, the intensity of anti-EMMPRIN/CD147 staining was not associated with other parameters. Therefore, EMMPRIN/CD147 was expressed in the majority of human osteosarcoma tissues and the relative levels of EMMPRIN/CD147 expression were associated positively with the pathogenic degree in human osteosarcoma.

High levels of EMMPRIN/CD147 expression are associated negatively with the survival period of osteosarcoma patients. We followed up all patients for a mean period of 32 months (8-72 months) and found that 42 patients (42/55) succumbed to the osteosarcoma without other evident diseases during the follow-up period. The mean survival time for the patients with osteosarcoma at stage IIB/III 
A

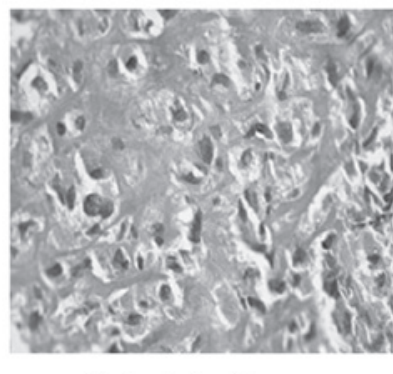

Osteoblastic

\section{B}

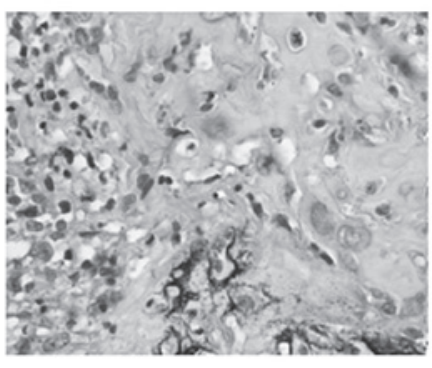

Chondroblastic

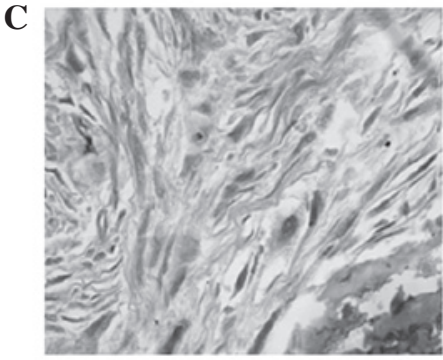

Fibroblastic

Figure 3. Histological characterization of osteosarcoma. Individual osteosarcoma specimens were characterized hisologically by hematoxylin and eosin staining. Data shown are representative images from each group of samples. (A) Osteoblastic, (B) Chondroblastic, (C) Fibroblastic.

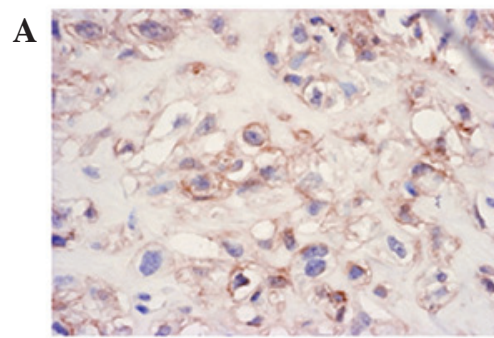

Osteosarcoma +

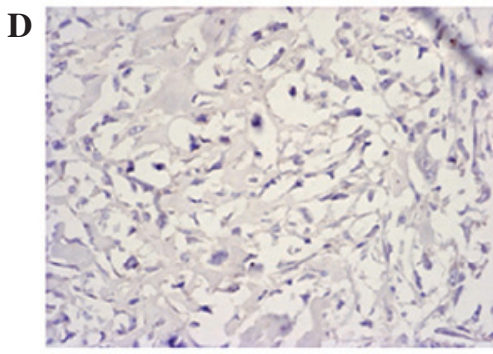

Osteosarcoma -

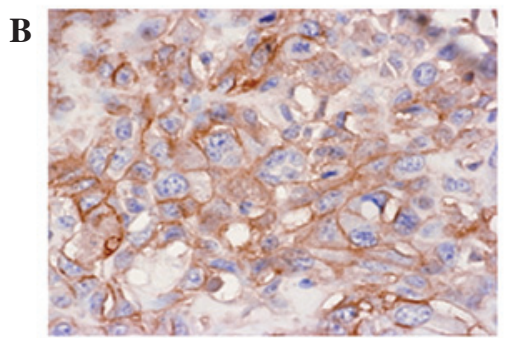

Osteosarcoma ++

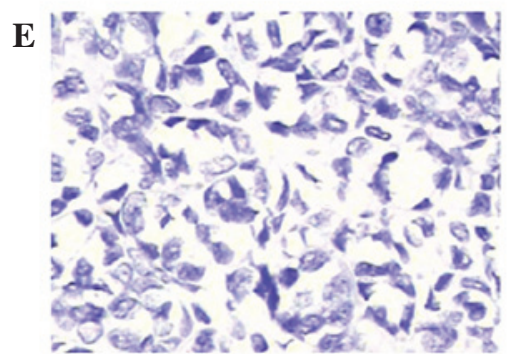

Osteosarcoma negative control

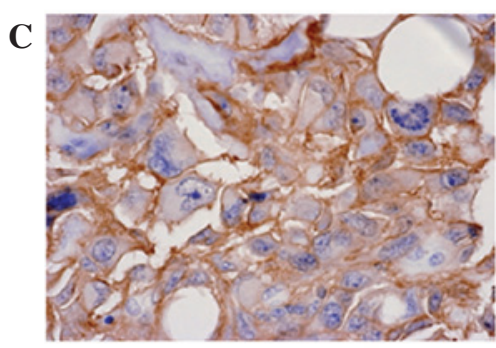

Osteosarcoma +++

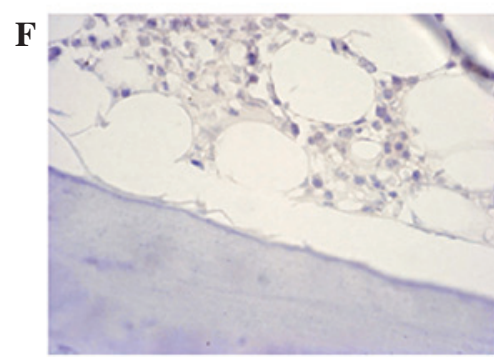

Non-tumor rib bone

Figure 4. Immunohistochemical analysis of EMMPRIN/CD147 expression in osteosarcoma tissues. Individual osteosarcoma tissue sections were subjected to immunohistochemistry analysis using anti-EMMPRIN/CD147 and DAB. The intensity of anti-EMMPRIN/CD147 staining was semi-quantitatively analyzed. Data shown are representative images from each group of samples. (A-C) The intensity of EMMPRIN/CD147 expression at (A) +, (B) ++ and (C) +++ in osteosarcoma. (D) Negative-staining osteosarcoma. (E) Negative controls using PBS to replace the anti-EMMPRIN/CD147 primary antibody. (F) Non-tumor rib bone.

was 33 months, which was significantly shorter than that of patients with tumors at stage IIA $(\mathrm{P}<0.05)$. A multivariate analysis (Cox regression model) revealed that the tumor pathological degree (IIA vs. IIB/III) $(\mathrm{P}=0.001 ; 95 \% \mathrm{CI}$, 5.004-85.535) and EMMPRIN/CD147 expression ( $\mathrm{P}=0.002$; 95\% CI, 1.810-13.238), but not gender, age, tumor location, treatment or histological subtype, were significantly associated with the overall survival (Fig. 5A; Table I). Notably, patients with osteosarcoma that had moderate/strong EMMPRIN/CD147 expression displayed significantly shorter periods of overall survival, compared with those with negative/weak EMMPRIN/CD147-expressing tumors (Fig. 5A; Table I; $\mathrm{P}<0.05)$. A similar pattern of event-free survival was observed in these two groups of patients (Fig. 5B). Therefore, the expression of EMMPRIN/CD147 is likely to be a negative prognostic factor for the survival of patients with osteosarcoma.

\section{Discussion}

Osteosarcoma is the most common primary malignant bone tumor in children and adolescents, and has an extremely poor prognosis in patients due to fast metastasis and tumor recurrence (2-6), although significant improvements in the prognosis of localized osteosarcoma have been noted over the past 30 years $(3,6)$. We found that 42 out of 55 patients succumbed to osteosarcoma during the 5-year follow-up, supporting the notion that osteosarcoma has an extremely poor prognosis in patients. The value of currently used prognostic factors, including chemotherapy response, tumor volume, older age, gender and p-glycoprotein expression remains in debate. Our data demonstrated that EMMPRIN/CD147 was expressed in human osteosarcoma cell lines and osteosarcoma tissues. The relative levels of EMMPRIN/CD147 expression in human osteosarcoma tissues were positively correlated 
A

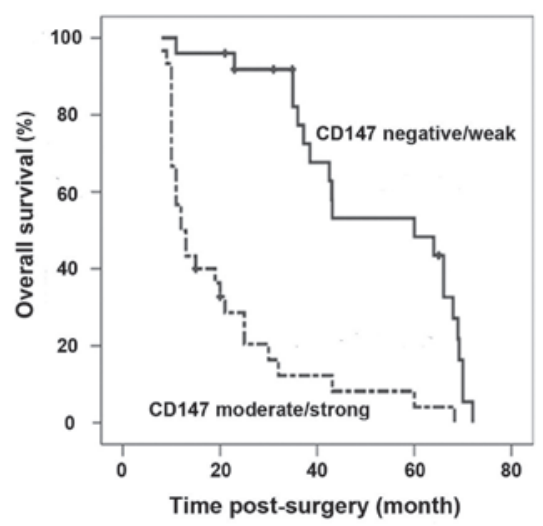

B

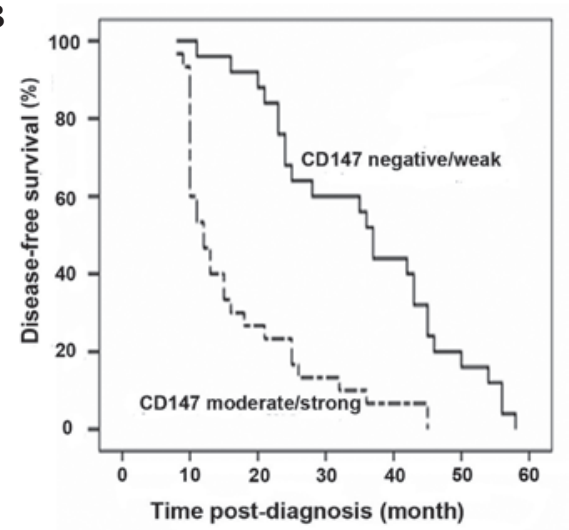

Figure 5. Survival curves of patients. The survival time of individual patients was determined and the survival curves of patients with differential expression of EMMPRIN/CD147 were generated, according to the Kaplan-Meier method. (A) Overall survival of patients with osteosarcoma. (B) Event-free survival of patients with osteosarcoma.

with the pathological degree, but negatively correlated with the survival period. Therefore, our findings suggest that the levels of EMMPRIN/CD147 expression may be used as one prognostic factor for estimating the survival of osteosarcoma patients in clinics.

EMMPRIN/CD147 is a highly glycosylated transmembrane protein belonging to the immunoglobulin superfamily 34 and is widely presented and/or overexpressed on the membrane surface of various malignant tumor cells $(7,8,10-17)$. Due to its close association with pathological classification and overall survival analysis, EMMPRIN/CD147 has been potentially suggested as a prognostic factor in certain types of malignant tumors $(7,8,10,12-15,17,21-24)$. In the present study, our results indicated that EMMPRIN/CD147 was expressed not only in all three human osteosarcoma cell lines, but also in the cell membrance and cytoplasm of osteosarcoma tissues from the majority of patients pathologically diagnosed with osteosarcoma at grade IIA or above. These results indicated that EMMPRIN/CD147 may be one of the prognostic factors for human osteosarcoma. In addition, our data indicated that the levels of EMMPRIN/CD147 expression were significantly associated with the pathological degree of osteosarcoma, but not with age, tumor size, location, gender, treatment or histological subtype. These findings are consistent with a recent study (33) and further support the notion that the pathological stage and EMMPRIN/CD147 expression may be ideal prognostic factors for human osteosarcoma.

Our findings indicate that the expression of EMMPRIN/CD147 was closely associated with the pathological degree of osteosarcoma, suggesting that EMMPRIN/CD147 may be significant in the development and progression of osteosarcoma. Previous studies have shown that EMMPRIN/CD147, through multiple pathways and mechanisms, stimulates adjacent fibroblasts to produce matrix metalloproteinases, and thus promotes survival, invasion and metastasis of tumor cells $(13,18-23,36)$. Indeed, EMMPRIN/CD147 expression is correlated significantly with the stage of clinicopathology in thyroid carcinoma (9), adenocarcinomas (7), esophageal squamous cell carcinomas (37) and prostate cancer (14). Furthermore, treatment with anti-EMMPRIN/CD147 antibody delays the formation of tumors in animal models (38). In addition, treatment with EMMPRIN/CD147-specific RNA interference inhibits the tumorigenicity and metastasis of human lymphoid neoplasms, oral squamous carcinoma, prostate carcinoma and bladder carcinoma cells, increasing their sensitivity to chemotherapeutic drugs $(8,39,40)$. These findings have indicated that RMMPRIN/CD147 may be an important molecule in tumor progression and an attractive target for antitumor treatment. Hence, EMMPRIN/CD147 may be one therapeutic candidate target for the treatment of osteosarcoma in clinics.

In conclusion, our data indicated that high levels of EMMPRIN/CD147 were expressed in human osteosarcoma cells and tissues. The levels of EMMPRIN/CD147 expression were positively correlated with the clinicopathological degree of osteosarcoma and negatively correlated with the survival of osteosarcoma patients. Therefore, EMMPRIN/CD147 expression may be used as a potential prognostic marker and therapeutic target for the intervention of human osteosarcoma. We recognize the limitation of the small sample size used in the present study and advise that further studies of the dynamic expression of EMMPRIN/CD147 with a bigger population of osteosarcoma patients are warranted.

\section{Acknowledgements}

The authors would like to acknowledge Medjaden for their great help in preparing the manuscript.

\section{References}

1. Clark JC, Dass CR and Choong PF: A review of clinical and molecular prognostic factors in osteosarcoma. J Cancer Res Clin Oncol 134: 281-297, 2008.

2. Davicioni E, Wai DH and Anderson MJ: Diagnostic and prognostic sarcoma signatures. Mol Diagn Ther 12: 359-374, 2008.

3. Fagioli F, Biasin E, Mereuta OM, et al: Poor prognosis osteosarcoma: new therapeutic approach. Bone Marrow Transplant 41 (Suppl 2): S131-S134, 2008.

4. Sakamoto A and Iwamoto Y: Current status and perspectives regarding the treatment of osteo-sarcoma: chemotherapy. Rev Recent Clin Trials 3: 228-231, 2008.

5. Wang LL: Biology of osteogenic sarcoma. Cancer J 11: 294-305, 2005.

6. Federman N, Bernthal N, Eilber FC and Tap WD: The multidisciplinary management of osteosarcoma. Curr Treat Options Oncol 10: 82-93, 2009.

7. Hakuma N, Betsuyaku T, Kinoshita I, et al: High incidence of extracellular matrix metalloproteinase inducer expression in non-small cell lung cancers. Association with clinicopathological parameters. Oncology 72: 197-204, 2007. 
8. Jia L, Wei W, Cao J, Xu H, Miao X and Zhang J: Silencing CD147 inhibits tumor progression and increases chemosensitivity in murine lymphoid neoplasm P388D1 cells. Ann Hematol 88 753-760, 2009

9. Aratake Y, Marutsuka K, Kiyoyama K, et al: EMMPRIN (CD147) expression and differentiation of papillary thyroid carcinoma: implications for immunocytochemistry in FNA cytology. Cytopathology 21: 103-110, 2009.

10. Chen T and Zhu J: Evaluation of EMMPRIN and MMP-2 in the prognosis of primary cutaneous malignant melanoma. Med Oncol 27: 1185-1191, 2009.

11. Zhang C, Tu Z, Du S, Wang Y and Wang Q: Expression of matrix metalloproteinase 2 and extracellular matrix metalloproteinase inducer are unfavorable postoperative prognostic factors in intrahepatic cholangiocarcinoma. Pathol Oncol Res 16: 47-53, 2010.

12. Buergy D, Fuchs T, Kambakamba P, et al: Prognostic impact of extracellular matrix metalloprotease inducer: immunohistochemical analyses of colorectal tumors and immunocytochemical screening of disseminated tumor cells in bone marrow from patients with gastrointestinal cancer. Cancer 115: 4667-4678, 2009.

13. Liang YX, He HC, Han ZD, et al: CD147 and VEGF expression in advanced renal cell carcinoma and their prognostic value. Cancer Invest 27: 788-793, 2009.

14. Han ZD, Bi XC, Qin WJ, et al: CD147 expression indicates unfavourable prognosis in prostate cancer. Pathol Oncol Res 15: 369-374, 2009.

15. Ju XZ, Yang JM, Zhou XY, Li ZT and Wu XH: EMMPRIN expression as a prognostic factor in radiotherapy of cervical cancer. Clin Cancer Res 14: 494-501, 2008.

16. Sillanpaa S, Anttila M, Suhonen K, et al: Prognostic significance of extracellular matrix metalloproteinase inducer and matrix metalloproteinase 2 in epithelial ovarian cancer. Tumour Biol 28 : 280-289, 2007.

17. Yang JM, O'Neill P, Jin W, et al: Extracellular matrix metalloproteinase inducer (CD147) confers resistance of breast cancer cells to anoikis through inhibition of Bim. J Biol Chem 281: 9719-9727, 2006.

18. Zhu H, Evans B, O'Neill P, et al: A role for $\mathrm{p} 53$ in the regulation of extracellular matrix metalloproteinase inducer in human cancer cells. Cancer Biol Ther 8: 1722-1728, 2009.

19. Bougatef F, Quemener C, Kellouche $S$, et al: EMMPRIN promotes angiogenesis through hypoxia-inducible factor-2alpha-mediated regulation of soluble VEGF isoforms and their receptor VEGFR-2. Blood 114: 5547-5556, 2009.

20. Tang Y, Nakada MT, Kesavan P, et al: Extracellular matrix metalloproteinase inducer stimulates tumor angiogenesis by elevating vascular endothelial cell growth factor and matrix metalloproteinases. Cancer Res 65: 3193-3199, 2005.

21. Zheng HC, Takahashi H, Murai Y, et al: Upregulated EMMPRIN/CD147 might contribute to growth and angiogenesis of gastric carcinoma: a good marker for local invasion and prognosis. Br J Cancer 95: 1371-1378, 2006.

22. Duivenvoorden WC, Hirte HW and Singh G: Transforming growth factor betal acts as an inducer of matrix metalloproteinase expression and activity in human bone-metastasizing cancer cells. Clin Exp Metastasis 17: 27-34, 1999.

23. Quemener C, Gabison EE, Naimi B, et al: Extracellular matrix metalloproteinase inducer up-regulates the urokinase-type plasminogen activator system promoting tumor cell invasion. Cancer Res 67: 9-15, 2007.
24. Riethdorf S, Reimers N, Assmann V, et al: High incidence of EMMPRIN expression in human tumors. Int J Cancer 119: 1800-1810, 2006

25. Wang $\mathrm{P}, \mathrm{Xu}$ TY, Guan YF, Su DF, Fan GR and Miao CY: Perivascular adipose tissue-derived visfatin is a vascular smooth muscle cell growth factor: role of nicotinamide mononucleotide. Cardiovasc Res 81: 370-380, 2009.

26. Wang P, Xu TY, Guan YF, et al: Nicotinamide phosphoribosyltransferase protects against ischemic stroke through SIRT1-dependent adenosine monophosphate-activated kinase pathway. Ann Neurol 69: 360-374, 2011.

27. Wang P, Guan YF, Du H, Zhai QW, Su DF and Miao CY: Induction of autophagy contributes to the neuroprotection of nicotinamide phosphoribosyltransferase in cerebral ischemia. Autophagy 8: 77-87, 2012.

28. Wang P, Zhang RY, Song J, et al: Loss of AMP-activated protein kinase-alpha2 impairs the insulin-sensitizing effect of calorie restriction in skeletal muscle. Diabetes 61: 1051-1061, 2012

29. Enneking WF, Spanier SS and Goodman MA: A system for the surgical staging of musculoskeletal sarcoma. Clin Orthop Relat Res 153: 106-120, 1980.

30. Enneking WF, Spanier SS and Goodman MA: Current concepts review. The surgical staging of musculoskeletal sarcoma. J Bone Joint Surg Am 62: 1027-1030, 1980.

31. Picci P, Bacci G, Campanacci M, et al: Histologic evaluation of necrosis in osteosarcoma induced by chemotherapy. Regional mapping of viable and nonviable tumor. Cancer 56: 1515-1521, 1985.

32. Wang P, Yang FJ, Du H, et al: Involvement of leptin receptor long isoform (LepRb)-STAT3 signaling pathway in brain fat massand obesity-associated (FTO) downregulation during energy restriction. Mol Med 17: 523-532, 2011.

33. Zhou Q, Zhu Y, Deng Z, Long H, Zhang S and Chen X: VEGF and EMMPRIN expression correlates with survival of patients with osteosarcoma. Surg Oncol 20: 13-19, 2011.

34. Madigan MC, Kingsley EA, Cozzi PJ, Delprado WJ, Russell PJ and $\mathrm{Li} \mathrm{Y}$ : The role of extracellular matrix metalloproteinase inducer protein in prostate cancer progression. Cancer Immunol Immunother 57: 1367-1379, 2008.

35. Ishibashi Y, Matsumoto T, Niwa M, et al: CD147 and matrix metalloproteinase-2 protein expression as significant prognostic factors in esophageal squamous cell carcinoma. Cancer 101: 1994-2000, 2004.

36. Nabeshima K, Iwasaki H, Koga K, Hojo H, Suzumiya J and Kikuchi M: Emmprin (basigin/CD147): matrix metalloproteinase modulator and multifunctional cell recognition molecule that plays a critical role in cancer progression. Pathol Int 56: 359-367, 2006.

37. Cheng MF, Tzao C, Tsai WC, et al: Expression of EMMPRIN and matriptase in esophageal squamous cell carcinoma: correlation with clinicopathological parameters. Dis Esophagus 19: 482-486, 2006.

38. Dean NR, Newman JR, Helman EE, et al: Anti-EMMPRIN monoclonal antibody as a novel agent for therapy of head and neck cancer. Clin Cancer Res 15: 4058-4065, 2009.

39. Kuang YH, Chen X, Su J, et al: RNA interference targeting the CD147 induces apoptosis of multi-drug resistant cancer cells related to XIAP depletion. Cancer Lett 276: 189-195, 2009.

40. Han ZD, He HC, Bi XC, et al: Expression and clinical significance of CD147 in genitourinary carcinomas. J Surg Res 160: 260-267, 2010. 